TWEPP 2016

26 - 30 SEP 2016

KARLSRUHE

\title{
Readout and Trigger for the AFP Detector at ATLAS Experiment
}

\section{Kocian on behalf of the ATLAS Collaboration}

SLAC, 2575 Sand Hill Road, Menlo Park, USA

E-mail: kocian@slac.stanford.edu

Abstract: AFP, the ATLAS Forward Proton consists of silicon detectors at $205 \mathrm{~m}$ and $217 \mathrm{~m}$ on each side of ATLAS. In 2016 two detectors in one side were installed. The FEI4 chips are read at 160 Mbps over the optical fibers. The DAQ system uses a FPGA board with Artix chip and a mezzanine card with RCE data processing module based on a Zynq chip with ARM processor running ArchLinux. In this contribution we give an overview of the AFP detector with the commissioning steps taken to integrate with the ATLAS TDAQ. Furthermore first performance results are presented.

KeYwords: Data acquisition concepts, Trigger concepts and systems 


\section{Contents}

1 The AFP Experiment 1

2 Readout and Trigger 1

2.1 Readout 1

2.2 Trigger 2

3 DAQ System 2

$\begin{array}{lll}3.1 & \text { Hardware } & 2\end{array}$

3.2 Firmware 3

4 System Architecture $\quad 4$

5 Conclusion and Outlook 5

\section{The AFP Experiment}

The ATLAS Forward Proton detector [1] aims to measure the transfer momentum and energy loss of very forward protons colliding in the ATLAS [2] interaction point. For this purpose, the detector consists of two Roman Pots per ATLAS side at $205 \mathrm{~m}$ and $217 \mathrm{~m}$ from the IP as shown in figure 1, each of them containing a 3D silicon tracker [3] and a time-of-flight detector in the far stations.

A tracker is composed of four pixel modules arranged along the beamline. The modules consist of 3D sensors $2 \mathrm{~cm} \times 2 \mathrm{~cm}$ in size which are bump-bonded to FEI4B readout chips. Each module contains 26880 pixels of a size of $250 \mu \mathrm{m}$ x $50 \mu \mathrm{m}$. The pixel modules were developed for the ATLAS innermost pixel layer (IBL) [4]. For data taking the sensors are moved into the beamline with the edge of the sensors separated from the beam by $20 \sigma$ of the beam size, corresponding to a distance of 4-8 $\mathrm{mm}$.

A first stage of installation took place during the LHC shutdown of 2015-2016. In that period, the two Roman Pot stations on one side were installed, together with a silicon tracker in each station (AFP0+2) in February 2016. In the LHC winter shutdown of 2016-2017 the second arm of AFP will be installed, as well as the time-of-flight-counters that will be used for background rejection.

\section{Readout and Trigger}

\subsection{Readout}

The frontend chips generate $8 \mathrm{~b} 10 \mathrm{~b}$ encoded serial data streams at $160 \mathrm{Mbps}$. The first portion of the transmission line consists of an $8 \mathrm{~m}$ long twisted pair cable. At the end of the cable the electrical signal is converted into an optical signal and transmitted over a $200 \mathrm{~m}$ long optical MTP fiber 


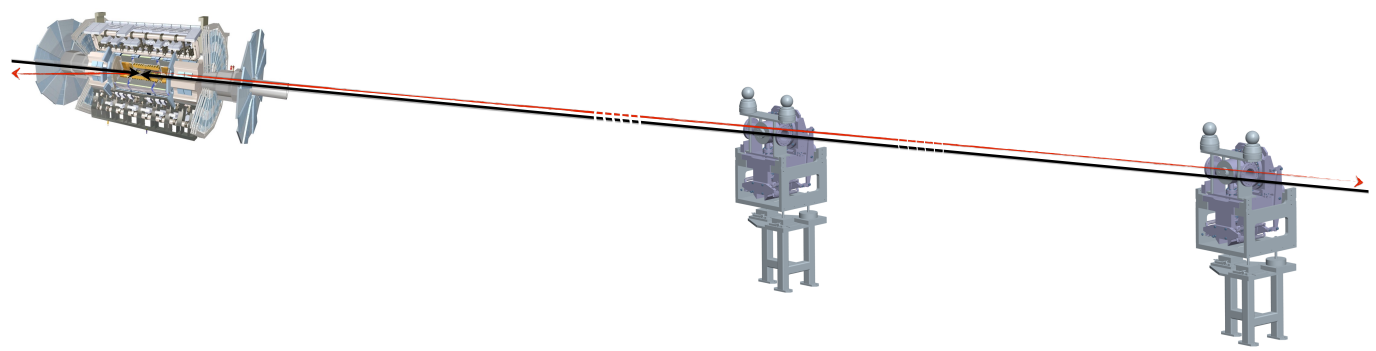

Figure 1. Schematic view of one arm of the AFP detector.

ribbon. For the downlink TTC information from ATLAS is transmitted to the frontend chips as a biphasemark encoded bitstream at $80 \mathrm{Mbps}$. Each TTC line is shared between two chips which can be addressed individually. On the detector side of the $200 \mathrm{~m}$ long fiber the optical converter board (developed for the ATLAS pixel detector) also decodes this bitstream and sends a $40 \mathrm{MHz}$ clock and a $40 \mathrm{Mbps}$ command bitstream to the frontends over the $8 \mathrm{~m}$ long twisted pair cable. The cable connects to the local trigger board (LTB) which distributes the signals to the frontends over short flat ribbon cables. An ASIC on the LTB (hitbus chip) applies additional, individual fine delays to the clock and command lines in a range from 0 to 8 ns. There is one LTB per Roman Pot station. In the counting house the signals are received and transmitted using commercial Snap-12 fiber modules. In total there are two receivers and one transmitter in the current system.

\subsection{Trigger}

The OR of the discriminated signals from all enabled pixels of each frontend chip is sent to an ASIC (hitbus chip) located on the LTB. The hitbus chip can combine three of the four trigger signals logically as OR, AND, or majority vote. The combined signal is synchronized with the LHC clock inside the hitbus chip and then sent to the counting house over an aircore coaxial cable in NIM logic. The high propagation speed in the aircore cable ensures that the trigger signal from AFP complies with the standard ATLAS trigger latency.

At the counting house the signal is split. One copy goes to the ATLAS Central Trigger Processor for inclusion of the AFP trigger in standard ATLAS runs while the other copy goes to a separate TTC crate which allows for standalone operation of AFP independent of the current state of ATLAS TDAQ.

\section{DAQ System}

\subsection{Hardware}

The data acquisition board used for AFP is the HSIO-II shown in figure 2. It was developed at SLAC and is part of the RCE DAQ system family [5]. It contains a Xilinx Artix 200 FPGA which 


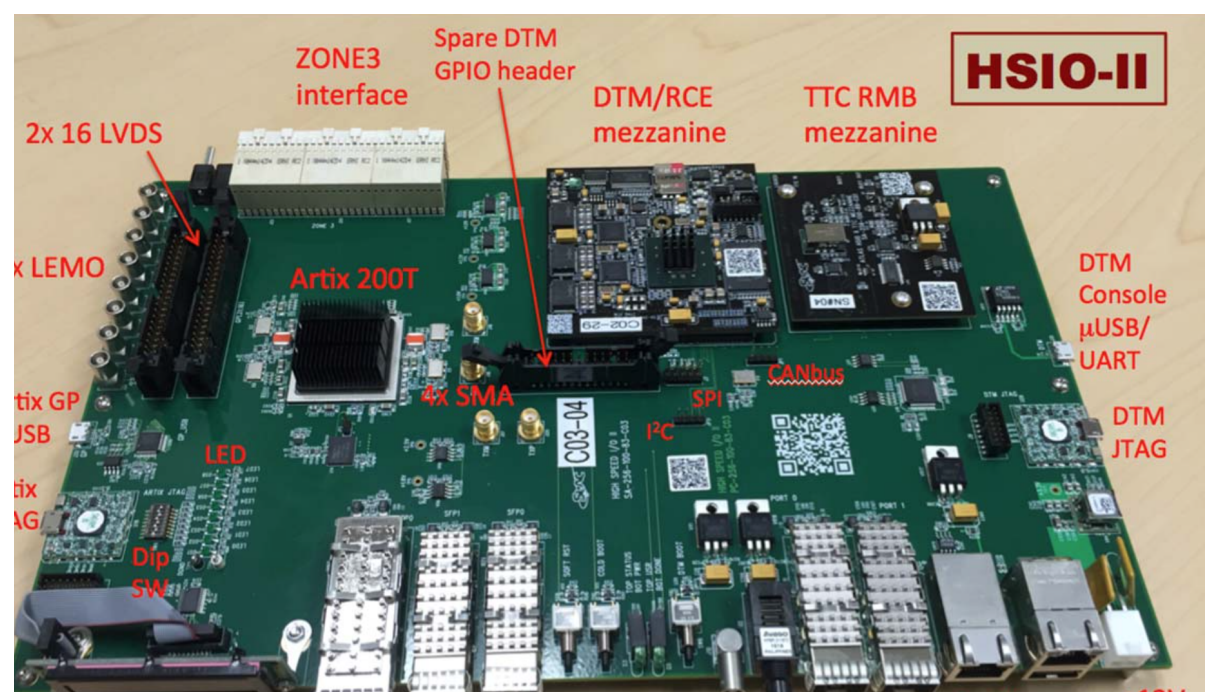

Figure 2. The HSIO-II DAQ board.

implements most of the DAQ functionality. The HSIO has an interface to the ATLAS TTC distribution system. Clock-data-recovery of the TTC signal is done on a mezzanine board on the HSIO. Another mezzanine board contains a Reconfigurable Cluster Element (RCE) with a system-on-chip which is based on the Xilinx Zynq Z7030. The Zynq contains a dual core ARM CPU running ArchLinux which has a DMA interface with the FPGA fabric and two ethernet interfaces. The RCE has 1 Gigabyte of RAM. It communicates with the Artix on the HSIO over a high-speed line at 3.125 Gbps using a custom protocol called PGP.

The processor configures the frontend chips, runs calibration scans, and monitors the firmware during data taking.

The Snap-12 fiber modules are located on an adapter board that plugs into the back of the HSIO via a 240 pin ATCA zone-3 connector. For lab testing an electrical adapter board can be used instead.

\subsection{Firmware}

The firmware on the Artix FPGA on the HSIO implements the following functions illustrated in figure 3:

- Serialization and deserialization of the frontend data.

- Communication with the RCE.

- Decoding of the TTC bitstream.

- Communication with the Readout Subsystem (ROS) through the S-Link protocol.

- Event fragment building. 
Communication with the RCE is realized via the custom PGP protocol. This protocol allows for shipping of data frames in both directions, register access on the Artix FPGA from the RCE, and sending of command opcodes. The registers on the HSIO are used to configure the firmware. The data downlink is used to configure the frontend chips while the data uplink sends the frontend data fragments to the RCE when running in standalone calibration mode. During data taking the data is streamed directly to the ROS through an SFP module on the HSIO. There is also a debugging mode in which a copy of the event fragment is sent to the RCE via PGP which allows for filtering and checking of fragments directly on the RCE.

The clock and trigger signals as well as two synchronization signals - bunch counter reset (BCR) and event counter reset (ECR) - are decoded from the TTC data stream that the HSIO receives over a fiber. The clock is distributed to the frontends. An internal clock can be used when the LHC clock is not available. The firmware also generates a "busy" signal when a FIFO for frontend data or for TTC data reaches a level where it is $80 \%$ full. The "busy" signal is output via a LEMO connector at the front of the HSIO.

The event fragment builder combines the data from all frontends for each trigger into a single data fragment. When it receives a trigger from the TTC decoder it reads the data from the FIFOs into which the frontend data is written. It also checks the frontend data for consistency of level-1 id and bunch crossing id and flags inconsistent data in the fragment. In case of a continuous mismatch the data from a frontend will be ignored and the FIFO will be reset at the next LHC event counter reset. If a frontend does not send any data for two consecutive triggers then the fragment event builder assumes that there is a technical problem with the frontend, e.g. caused by a single event upset, and ignores data from the frontend for the rest of the run to avoid high deadtimes from continuous timeouts. Another feature of the event fragment builder is that it decodes service messages from the frontends. If a frontend signals that it missed a trigger because its buffers were full then the event builder will take the missing data frame into account in its bookkeeping.

The event fragment builder generates a number of status words and computes occupancies by frontend. These can be read out by the RCE via PGP. The information is published to the ATLAS Information Service (IS).

\section{System Architecture}

ATLAS Run Control communicates with AFP via a rack mounted Linux server. This server has two ethernet interfaces: One connects to the ATLAS network, and a second one connects to the RCE on the HSIO board over a restricted network. Software on the server translates the run control transitions for the RCE. Communication with the RCE happens over Gigabit copper ethernet using Deltavsoft RCF software [6] for inter-process communication. The server also reads the configuration files for the frontends from NFS disk and transmits the file contents to the RCE.

For calibration scans a standalone GUI-based application is run on the server. Using this application the thresholds and feedback currents of the individual pixels can be determined and 


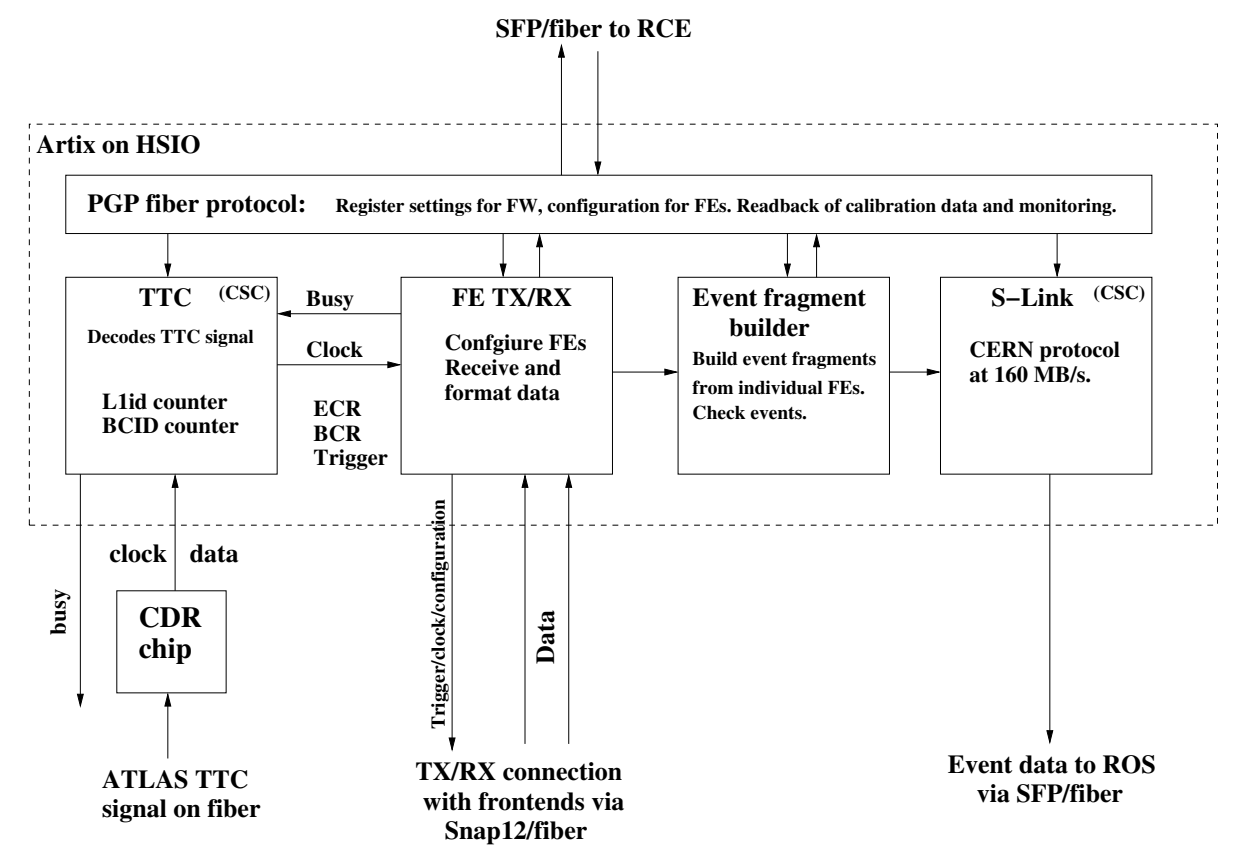

Figure 3. A schematic representation of the data acquisition firmware on the Artix FPGA.

configuration files with a uniform tuning can be generated.

\section{Conclusion and Outlook}

AFP had a successful start as a new detector in ATLAS this year. The AFP trigger and readout system has been performing smoothly and efficiently within the ATLAS framework. Figure 4 [7] shows two performance plots: On the left an occupancy hitmap for a single tracker module is shown, while the plot on the right shows the hit correlation between two consecutive modules in one dimension demonstrating that the DAQ processes and synchronizes the data correctly.

In the winter shutdown of 2016/2017 the AFP DAQ system will be upgraded to include the second arm of AFP as well as the readout of the time-of-flight detector. The standalone HSIO-II board will be replaced by an ATCA based RCE system. Readout of the frontends will no longer be done over single fibers at $160 \mathrm{Mbps}$. Instead, CERN's GBT chipset [8] will be used to multiplex the data from up to 20 frontends at $160 \mathrm{Mbps}$ onto a single $4.8 \mathrm{Gbps}$ fiber.

The current RCE software can be reused without modification. The firmware blocks will be reutilized and adapted to the GBT based system.

\section{References}

[1] ATLAS Forward Proton - Phase-I Upgrade, Technical Design Report, ATLAS-TDR-024-2015, 2015

[2] ATLAS Collaboration (Aad, G. et al.), The ATLAS Experiment at the CERN Large Hadron Collider, JINST 3 (2008) 03 

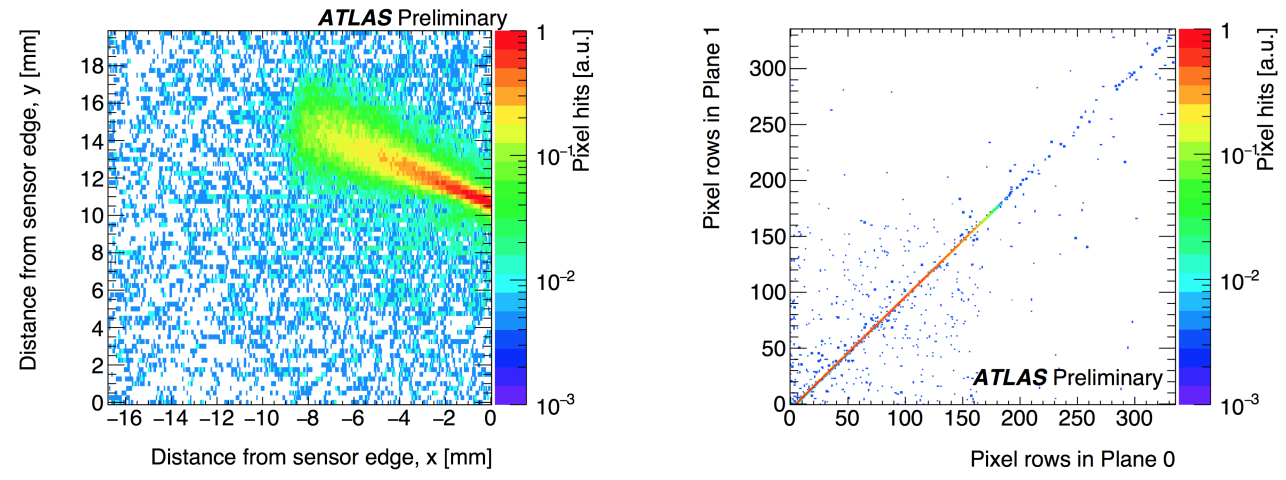

Figure 4. Left side: Hit occupancy in a single tracker module. Right side: Hit correlation between two consecutive tracker modules.

[3] S. Grinstein et al., Module production of the one-arm AFP 3D pixel tracker, submitted to JINST, November, 2016, arXiv: 1611.01005 [physics.ins-det].

[4] ATLAS Insertable B-Layer Technical Design Report, CERN-LHCC-2010-013, ATLAS-TDR-19, 2010

[5] https://indico.cern.ch/event/546846

[6] http://www.deltavsoft.com

[7] https://twiki.cern.ch/twiki/bin/view/AtlasPublic/ForwardDetPublicResults

[8] P. Moreira, The radiation hard GBTX link interface chip, in PH-ESE Electronics Seminars, November 26, CERN, Switzerland (2013). 